\title{
Physiological- and performance-related effects of acute olive oil supplementation at moderate exercise intensity
}

\author{
Laura Esquius ${ }^{1,2}$, Sergi Garcia-Retortillo ${ }^{3,4^{*}}$ (D), Natàlia Balagué ${ }^{4}$, Robert Hristovski ${ }^{5}$ and Casimiro Javierre ${ }^{2}$
}

\begin{abstract}
Background: The consumption of olive oil is associated with a diminished risk of cardiovascular disorders and mortality, but the impact of olive oil supplementation on endurance performance is still unclear. Since the beneficial effects of olive oil are observed at a systemic level, its effectiveness may not be precisely measured through the commonly registered maximal and threshold values of some physiological and performance parameters. In contrast, we suggest evaluating it through variables able to capture the coordinated behaviour of physiological systems. Thus, the aim of the current research was to assess the effect of an acute extra virgin olive oil supplementation on cardiorespiratory coordination (CRC) and performance, compared to palm oil.
\end{abstract}

Methods: Three separate effort test sessions were carried out separated by 7-day interval. During each session, participants $(n=7)$ repeated the same progressive and maximal walking test, but under different dietary supplementations in a randomized order: (1) olive oil, (2) palm oil, and (3) placebo. A principal component (PC) analysis of selected cardiovascular and cardiorespiratory variables was carried out to evaluate CRC. Eigenvalues of the first $P C\left(P C_{1}\right)$ and the loadings of the cardiorespiratory variables onto $P C_{1}$ were compared among dietary supplementations. In order to more accurately evaluate CRC, all the tests were divided into 3 equal sections, corresponding to low, moderate, and high exercise intensities, and the aforementioned procedure was repeated for each section in all the tests.

Results: Statistically significant differences were observed regarding $\mathrm{PC}_{1}$ eigenvalues among dietary supplementations $\left(X^{2}(8,2)=6.3 ; p=.04\right)$, only at moderate intensity exercise. Specifically, $P C_{1}$ eigenvalues were higher under olive oil compared to palm oil ( $2.63 \pm 0.51$ vs. $2.30 \pm 0.28 ; Z=2.03 ; p=.04 ; d=0.80)$ and placebo supplementations ( $2.63 \pm 0.51$ vs. $2.38 \pm 0.36 ; Z=2.20 ; p=.03 ; d=0.57)$.

Conclusions: Supplementation with extra virgin olive oil increased CRC during a progressive walking test at moderate intensity, although did not change performance and other physiological markers. CRC analysis appears as a sensitive tool to investigate the physiological and performance effects of dietary supplementations.

Keywords: Cardiorespiratory coordination, Olive oil, Polyphenols, Unsaturated fatty acids, Dietary supplementation, Cardiorespiratory exercise testing, Complex adaptive systems, Principal components analysis

\footnotetext{
* Correspondence: sgarciaretortillo@gmail.com

${ }^{3}$ Complex Systems in Sport, University School of Health and Sport (EUSES),

University of Girona, C. Francesc Macià 65, 17190 Salt, Spain

${ }^{4}$ Complex Systems in Sport, Institut Nacional d'Educació Física de Catalunya

(INEFC), Universitat de Barcelona, Avda. de I'Estadi 12-22, 08038 Barcelona,

Spain

Full list of author information is available at the end of the article
}

(c) The Author(s). 2019 Open Access This article is distributed under the terms of the Creative Commons Attribution 4.0 International License (http://creativecommons.org/licenses/by/4.0/), which permits unrestricted use, distribution, and reproduction in any medium, provided you give appropriate credit to the original author(s) and the source, provide a link to the Creative Commons license, and indicate if changes were made. The Creative Commons Public Domain Dedication waiver (http://creativecommons.org/publicdomain/zero/1.0/) applies to the data made available in this article, unless otherwise stated. 


\section{Introduction}

Olive oil is the main cornerstone of the Mediterranean diet and its consumption, specifically the extra virgin variety, is associated with a reduced inflammation and a diminished risk of cardiovascular disorders and mortality [1-3]. These benefits may be related to its source of polyphenols, which have been shown to possess antimicrobial, antioxidant and anti-inflammatory systemic properties $[4,5]$. Despite the large amount of beneficial effects on health, the impact of olive oil supplementation on endurance performance is still unclear [6]. These effects have been measured through specific aerobic physiological markers like maximal oxygen uptake $\left(\mathrm{VO}_{2 \max }\right)$, but not according to variables able to capture the dynamic interactions among physiological systems.

On the basis that diets high in unsaturated fatty acids and endurance exercise share different positive effects on metabolic and cardiovascular health, and given that both seem to increase fat oxidative capacity, earlier research hypothesised that their combination may have synergic effects [7]. The main results revealed that unsaturated fatty acids supplementation tended to slightly increase fat oxidation after training compared to control conditions. However, these changes were not reflected in $\mathrm{VO}_{2 \max }$ or in other performance and physiological parameters [6-8]. Moreover, regarding the antioxidant effect of beverages containing polyphenols on physical performance and physiological markers, the results are not yet entirely clear, with studies reporting controversial effects [9]. These unclear results suggest that $\mathrm{VO}_{2 \max }$ and other commonly registered physiological and performance variables might not be sensitive enough to detect specific exercise-related changes as has been suggested by other authors [10-12]. A feasible explanation for the lack of sensitivity may stem from the fact that they provide little information on the nature of the dynamic interactions between physiological systems and their common role in an integrated network [13, 14]. More specifically, cardiovascular and respiratory systems change their interaction as a consequence of exercise [10]. Therefore, to capture such specific interactions, the time series analysis and the detection of coordinative variables [15] seems a recommendable strategy. These research approaches can detect not only quantitative differences related to maximal physiological values (e.g., $\mathrm{VO}_{2 \max }$ ), but also qualitative changes related to the coordinated activity among physiological systems, and their changes under exercise-related constraints $[16$, 17]. This coordinative changes occurring at systemic level as a consequence of exercise, although much less studied than those occurring at cellular or subcellular level, are not less relevant.

Since the anti-inflammatory and antioxidant effects of olive oil are observed at a systemic level [5], and its effectiveness may not be precisely measured through the commonly registered physiological and performance parameters, we proposed here to use a recently investigated coordinative variable (cardiorespiratory coordina tion; CRC) which has shown a higher responsiveness to training [10] and workload accumulation [11], in contrast to $\mathrm{VO}_{2 \max }$ and other markers of aerobic fitness. $\mathrm{CRC}$ is a novel variable informing about the co-variation of cardiovascular and respiratory variables during cardiorespiratory exercise testing $[10,11]$. It is estimated through principal components analysis (PCA), performed on the time series of selected variables. In contrast to isolated cardiorespiratory outcomes, the cardiorespiratory response to exercise can be represented through PCA, i.e., by a short set of principal components (PCs) extracted in decreasing order of importance (the first PC accounting for most of the variation). Principal components represent the maximum possible fraction of the variability from the original data, so that the total number of PCs reflects the degree of coordination among the selected variables. As pointed out by Kelso [18], a dimension reduction is a hallmark of formation of coordinative structures, and so, the decrease in the number of PCs and/or the increase in PC eigenvalues can be interpreted as an improvement in the efficiency of CRC (see Balagué et al. [10] for a detailed explanation).

Accordingly, the aim of the current research was to assess the effect of an acute fatty acid supplementation, in the form of extra virgin olive oil rich in polyphenols, on $\mathrm{CRC}$ and physiological and performance variables, compared to palm oil rich in unsaturated fatty acids and without polyphenols. We hypothesized that the positive effects of olive oil supplementation would be mainly reflected on CRC.

\section{Material and methods Participants}

To determine the sample size for this study a power analysis was conducted using G*Power 3.1. [19]. Similar studies of CRC during exercise [11] have reported moderate and large effect sizes. Using an effect size of $d=$ $0.75, \alpha<.05$, power $(1-\beta)=.95$, a minimum sample size of seven participants was established. Thus, seven healthy males (age $32.2 \pm 4.3$; height $180 \pm 5.4 \mathrm{~cm}$; body mass $74.9 \pm 7.7 \mathrm{Kg}$; body mass index $23.1 \pm 1.7 \mathrm{Kg} . \mathrm{m}^{-2}$ ), who were active runners competing at national level for at least 5 years and engaged in endurance activities between four and seven times per week (10-14 h), volunteered to participate in the study. Exclusion criteria consisted of: a) current or previous injury against exercise testing, taking fish oil or other fatty acids supplements, and c) any other conditions that may prevent the performance of a maximal exercise protocol. The experiment was approved by the local ethical committee and 
carried out according to the Helsinki Declaration. Participants read the study's description and risks and signed an informed consent before taking part in the study.

\section{Intervention and procedure Study design}

In this randomized cross-over controlled double-blind trial, three separate effort test sessions were carried out separated by 7-day intervals. During each session, participants repeated the same maximal walking test, but under different supplementations: (1) olive oil, (2) palm oil, and (3) placebo. Supplementation in the form of a gel at a temperature of about $10^{\circ} \mathrm{C}$ was orally administered blind to each participant. The testing order was randomized using a random number generator. On all visits participants reported to the laboratory in a similar postprandial state ( $3 \mathrm{~h}$ after a light meal), and were instructed not to perform any vigorous physical activity for $72 \mathrm{~h}$ before testing. The ratio of the light meal was approximately $70 \%$ carbohydrates, 15\% lipids, and 15\% proteins. The following breakfast suggestion was provided: orange juice $(250 \mathrm{~g})$, whole milk (250 g), cereals $(70 \mathrm{~g})$. Participants were requested to eat the same light meal before each test session and not change the diet during the study period.

\section{Maximal walking test}

A maximal walking test ensuring high overall energy cost, and lasting longer time on Fat max $_{\text {zone was se- }}$ lected. The test (Quasar, HP cosmos sports \& medical gmbh, Nussdorf-Traunstein, Germany) was conducted at a constant velocity of $6.5 \mathrm{Km} / \mathrm{h}$ and the slope was increased by $1 \%$ every $4 \mathrm{~min}$. Once the treadmill slope reached $20 \%$, the velocity was increased by $1 \mathrm{Km} / \mathrm{h}$ every 4 min until volitional exhaustion. During the test, participants breathed through a valve (Hans Rudolph, 2700, Kansas City, MO, USA) and respiratory gas exchange was determined through an automated open-circuit device (Metasys, Brainware, La Valette, France). Oxygen and $\mathrm{CO}_{2}$ content and air flow rate were monitored every $30 \mathrm{~s}$. Prior to each test, the system was calibrated with the following mixture: $\mathrm{O}_{2} 15 \%, \mathrm{CO}_{2} 5 \%, \mathrm{~N}_{2}$ balanced (Carburos Metálicos, Barcelona, Spain), as well as with ambient air. Electrocardiogram (ECG) was continually recorded (CardioScan v.4.0, DM Software, Stateline, Nevada, USA) to monitor heart rate (HR). All tests were carried out in a well-ventilated lab; the place temperature was $23^{\circ} \mathrm{C}$ and the relative humidity 48\%, with changes of no more than $1{ }^{\circ} \mathrm{C}$ in temperature and $10 \%$ in relative humidity.

\section{Supplements}

The active and placebo supplements were prepared using orange juice, with added modified starch to achieve a gel texture; the active A supplement also included extra virgin olive oil, and the Active B also included palm oil (see Table 1). Orange juice was used to obtain a nice taste in all the supplements, and to mask the taste of both olive and palm oil actives, achieving the same taste for all the supplements. The calorific value and fat content of the active supplements were much higher than in the placebo. The supplement was taken in the lab, $1 \mathrm{~h}$ before the beginning of the test. A $25 \mathrm{ml}$ dose of extra virgin olive oil was selected since it does not significantly induce a postprandial lipemia or an increase in oxidation markers in vivo, while a dose of 50 $\mathrm{ml}$ induces it [20].

\section{Data analysis}

\section{Cardiorespiratory coordination}

To study CRC, a principal components analysis (PCA) was carried out on the time series of selected cardiorespiratory variables in all tests and participants: expired fraction of $\mathrm{O}_{2}\left(\mathrm{FeO}_{2}\right)$, expired fraction of $\mathrm{CO}_{2}\left(\mathrm{FeCO}_{2}\right)$, ventilation (VE), and HR. Oxygen pulse, oxygen consumption, respiratory equivalents, respiratory exchange ratio, and other frequently recorded parameters during cardiorespiratory testing were not included in the PCA given their known deterministic mathematical relation with the aforesaid variables [11]. Continuous blood pressure monitoring could not be provided in this study. However, non-published results of our lab have shown similar results while analysing CRC with and without continuous blood pressure measurement. Bartlett's sphericity test and the KMO (Kaiser-Mayer-Olkin [21]) index were computed in order to evaluate the suitability of the application of the PCA. The number of PCs was defined by the Kaiser-Gutmann criterion, which recognizes PCs with eigenvalues $\lambda \geq 1.00$ as a significant [22]. Eigenvalues of the first $\mathrm{PC}\left(\mathrm{PC}_{1}\right)$ and the loadings of selected cardiorespiratory variables onto $\mathrm{PC}_{1}$ were compared among dietary supplementations by means of a Freedman ANOVA test and a Wilcoxon matched pairs test, since $\mathrm{PC}_{1}$ contains the largest amount of the data variance. Statistical analyses were completed using SPSS (v.23, SPSS Inc., USA).

In order to more accurately study $\mathrm{CRC}$, all tests were divided into three equal time sections (i.e., total exercising time / 3). The first, second, and third sections corresponded to low, moderate, and high exercise intensity, respectively. The cutting points between low to moderate, and moderate to high intensities were located at $40.29 \pm 10.57$ and $70.81 \pm 7.08 \% \mathrm{VO}_{2 \text { peak }}$, respectively. The aforementioned procedure was repeated for each section in all the tests. The total number of PCs and $\mathrm{PC}_{1}$ eigenvalues reflects the dimensionality or degree of cardiorespiratory coordination (higher numbers of PCs and/or lower $\mathrm{PC}_{1}$ eigenvalues entail lower coordination, 
Table 1 Supplement ingredients and nutritional content

\begin{tabular}{llll}
\hline & Active A & Active B & Placebo \\
\hline Ingredients & $100 \mathrm{ml}$ orange juice & $100 \mathrm{ml}$ orange juice & $100 \mathrm{ml}$ orange juice \\
& $25 \mathrm{ml}$ Extra-virgin olive oil & $25 \mathrm{ml}$ Palm oil & $8 \mathrm{~g}$ Modified starch \\
& $8 \mathrm{~g}$ Modified starch & $8 \mathrm{~g}$ Modified starch & 52.8 \\
Energy (Kcal) & 277 & 277 & 0.1 \\
Lipids (g) & 25 & 25 & 12.6 \\
Carbohydrates (g) & 12.6 & 12.6 & \\
\hline
\end{tabular}

whereas lower numbers of PCs and/or higher $\mathrm{PC}_{1}$ eigenvalues imply higher coordination (for a detailed explanation see Balagué et al. [10] and Garcia-Retortillo et al.[11]).

\section{Physiological and performance variables}

Performance (measured through the exercising time) and maximal and threshold values of cardiorespiratory variables were recorded during each test. Aerobic (AT) and anaerobic (AnT) thresholds were obtained through $\mathrm{O}_{2}$ and $\mathrm{CO}_{2}$ respiratory equivalents method [23]. A repeated measures ANOVA with Bonferroni post-hoc test was used to compare values at AT, AnT, and maximal performance among dietary supplementations. Effect sizes (Cohen's $d$ ) were computed to demonstrate the magnitude of standardized mean differences and an alpha level was set at 0.05 for all statistical tests.

\section{Results}

\section{Cardiorespiratory coordination}

Bartlett's sphericity test $(p<.001)$ and the KMO index $(0.70 \pm 0.14)$ showed a good sampling adequacy. At low intensity, all participants showed $2 \mathrm{PCs}$ in the three dietary supplementations. While $\mathrm{PC}_{1}$ was formed by VE, $\mathrm{FeCO}_{2}$, and $\mathrm{HR}, \mathrm{PC}_{2}$ was mainly formed by $\mathrm{FeO}_{2}$ (see Table 2). No significant differences were found in $\mathrm{PC}_{1}$ eigenvalues among dietary supplementations at low intensity. Similar to the results at low intensity, at moderate intensity all participants showed 2 PCs in the three supplementations. However, Friedman ANOVA showed statistically significant differences regarding $\mathrm{PC}_{1}$ eigenvalues among dietary interventions $\left(\chi^{2}(8,2)=7 ; p=.03\right.$; see Table 2). Specifically, olive oil supplementation revealed higher $\mathrm{PC}_{1}$ eigenvalues compared to palm oil $(Z=2.03 ; p=.04 ; d=0.80)$ and placebo supplementations $(Z=2.20 ; p=.03 ; d=0.57)$, indicating more co-variation among selected cardiorespiratory variables at moderate intensity. Significant differences between dietary supplementations were only found in the VE projection $\left(\chi^{2}(8,2)=6.3 ; p=.04\right.$; see Table 2). Concretely, VE projection was significantly higher in olive oil supplementation compared to palm oil $(\mathrm{Z}=2.37 ; p=.02 ; d=4.71)$, but not compared to placebo $(Z=0.56 ; p=.60)$. No significant differences were found in $\mathrm{PC}_{1}$ eigenvalues between palm oil and placebo supplementations at moderate exercise intensity. Finally, all participants showed $2 \mathrm{PCs}$ at high intensity, whereas $\mathrm{PC}_{1}$ was loaded by $\mathrm{VE}, \mathrm{FeO}_{2}$, and $\mathrm{HR}, \mathrm{PC}_{2}$ was mainly formed by $\mathrm{FeCO}_{2}$. No significant differences were found in $\mathrm{PC}_{1}$ eigenvalues among dietary supplementations at high intensity.

Table 2 Means (standard deviations) of $\mathrm{PC}_{1}$ eigenvalues and projection of the selected cardiorespiratory variables onto $\mathrm{PC}_{1}$

\begin{tabular}{|c|c|c|c|c|c|c|c|c|c|}
\hline & \multicolumn{3}{|l|}{ 1st third } & \multicolumn{3}{|l|}{ 2nd third } & \multicolumn{3}{|l|}{ 3rd third } \\
\hline & Olive Oil & Palm Oil & Placebo & Olive Oil & Palm Oil & Placebo & Olive Oil & Palm Oil & Placebo \\
\hline \multirow[t]{2}{*}{$\overline{\mathrm{PC}_{1} \text { Eigenvalues }}$} & 2,47 & 2,43 & 2,53 & $2,63^{\mathrm{a}}$ & $2,30^{a}$ & $2,38^{a}$ & 2,48 & 2,42 & 2,59 \\
\hline & $(0,42)$ & $(0,58)$ & $(0,26)$ & $(0,51)$ & $(0,28)$ & $(0,36)$ & $(0,39)$ & $(0,32)$ & $(0,21)$ \\
\hline \multirow[t]{2}{*}{ Veprojection } & 0,86 & 0,82 & 0,84 & $0,91^{\mathrm{a}}$ & $0,79^{a}$ & $0,89^{a}$ & 0,83 & 0,92 & 0,96 \\
\hline & $(0,06)$ & $(0,13)$ & $(0,07)$ & $(0,03)$ & $(0,17)$ & $(0,05)$ & $(0,34)$ & $(0,06)$ & $(0,02)$ \\
\hline \multirow[t]{2}{*}{$\mathrm{FeO}_{2}$ projection } & 0,57 & 0,67 & 0,58 & 0,61 & 0,64 & 0,50 & 0,71 & 0,78 & 0,83 \\
\hline & $(0,29)$ & $(0,23)$ & $(0,20)$ & $(0,20)$ & $(0,30)$ & $(0,28)$ & $(0,31)$ & $(0,26)$ & $(0,17)$ \\
\hline \multirow[t]{2}{*}{$\mathrm{FeCO}_{2}$ projection } & 0,70 & 0,86 & 0,79 & 0,66 & 0,73 & 0,63 & 0,55 & 0,51 & 0,42 \\
\hline & $(0,66)$ & $(0,13)$ & $(0,31)$ & $(0,34)$ & $(0,17)$ & $(0,28)$ & $(0,18)$ & $(0,31)$ & $(0,26)$ \\
\hline \multirow[t]{2}{*}{ HRprojection } & 0,76 & 0,58 & 0,86 & 0,92 & 0,75 & 0,87 & 0,82 & 0,69 & 0,84 \\
\hline & $(0,07)$ & $(0,62)$ & $(0,32)$ & $(0,03)$ & $(0,20)$ & $(0,14)$ & $(0,21)$ & $(0,30)$ & $(0,15)$ \\
\hline
\end{tabular}

${ }^{\text {a }}$ statistically significant differences among dietary supplementations; $P C$ principal component, VE ventilation, FeO2 expired fraction of oxygen, FeCO2 expired fraction of carbon dioxide, $H R$ heart rate 


\section{Physiological and performance variables}

Table 3 shows AT (corresponding to moderate exercise intensity), AnT (corresponding to high exercise intensity), and maximal values of cardiorespiratory and performance (i.e., exercising time) variables in the three dietary supplementations. Non-statistically significant differences were found among supplementations. However, while specifically comparing VE, $\mathrm{PETO}_{2}$, and $\mathrm{PETCO}_{2}$ values corresponding to AT, olive oil revealed significantly lower (VE and $\mathrm{PETO}_{2}$ ) and significantly higher $\left(\mathrm{PETCO}_{2}\right)$ values compared to palm oil supplementation (see Table 3 and Fig. 1).

\section{Discussion}

The present research was conceived to assess the effect of an acute fatty acid supplementation, in the form of extra virgin olive oil rich in polyphenols, on CRC and performance, compared to palm oil and placebo. An increase in CRC under olive oil supplementation, probably provoked by its high content in polyphenols, was observed. However, these improvements were not reflected on the commonly evaluated maximal performance and physiological variables. These results suggest that CRC could be a sensitive tool to detect systemic effects linked to dietary supplementations.

The improvement in CRC under olive oil supplementation was solely observed at moderate intensity during the incremental test. Specifically, $\mathrm{PC}_{1}$ eigenvalues were significantly higher compared to palm oil and placebo supplementations. Since $\mathrm{PC}_{1}$ eigenvalues show the ratio of explained variance by $\mathrm{PC}_{1}[10]$, these results, indicating an increase in the degree of co-variation among the selected physiological variables, informed about an improvement in CRC. In other words, under olive oil supplementation, the ventilatory efficiency of the cardior espiratory system seemed larger. These results might be explained by the high content of olive oil in phenolic compounds, tocopherol, or carotenoids, which have been shown to possess antioxidant and anti-inflammatory properties, by producing beneficial effects on lipid oxidation and in general oxidative stress $[4,5]$. As pointed out by Sallam and Laher [24], acute bouts of exercise provoke transient damage to contracting skeletal muscles, triggering an inflammatory response that

Table 3 Means (standard deviations) of performance and physiological variables, under olive oil, palm oil, and placebo supplementations

\begin{tabular}{|c|c|c|c|c|c|}
\hline Variables & Values & Olive oil & Palm oil & Placebo & Statistical differences \\
\hline Exercising time (min) & Maximal & $77.3(2.0)$ & $76.3(2.3)$ & $77.4(2.3)$ & \\
\hline \multirow[t]{3}{*}{ Respiratory rate $\left(\mathrm{b} \cdot \mathrm{min}^{-1}\right)$} & AT & $31.6(1.1)$ & $31.4(1.8)$ & $32.7(1.6)$ & \\
\hline & AnT & $37.5(3.1)$ & $41.2(4.1)$ & $39.2(2.5)$ & \\
\hline & Maximal & $46.5(4.1)$ & $52.3(7.6)$ & $48.7(5.1)$ & \\
\hline \multirow[t]{3}{*}{ Ventilation $\left(L \cdot \mathrm{min}^{-1}\right)$} & AT & $53.3(4.6)$ & $58.7(4.7)$ & $57.4(5.5)$ & (1) $p=.03 ; d=0.80$ \\
\hline & AnT & $83.9(8.3)$ & $87.7(10.5)$ & $90.5(8.7)$ & \\
\hline & Maximal & $112.9(9.1)$ & $119.8(16.5)$ & $117.9(11.7)$ & \\
\hline \multirow[t]{3}{*}{ Oxygen uptake $\left(L \cdot \min ^{-1}\right)$} & AT & $2.15(0.16)$ & $2.31(0.20)$ & $2.36(0.24)$ & \\
\hline & AnT & $3.19(0.2)$ & $3.22(0.3)$ & $3.40(0.2)$ & \\
\hline & Peak & $3.85(0.1)$ & $3.96(0.2)$ & $4.00(0.2)$ & \\
\hline \multirow[t]{3}{*}{$\mathrm{PETO}_{2}(\mathrm{mmHg})$} & AT & $100.9(1.3)$ & $102.7(1.3)$ & $102.3(1.2)$ & (1) $p=.02 ; d=1.38$ \\
\hline & AnT & $103.8(2.2)$ & $105.3(2.7)$ & $104.2(1.9)$ & \\
\hline & Maximal & $108.6(2.7)$ & $109.6(3.5)$ & $108.8(2.8)$ & \\
\hline \multirow[t]{3}{*}{$\mathrm{PETCO}_{2}(\mathrm{mmHg})$} & AT & $42.1(0.9)$ & $40.1(0.8)$ & $40.9(0.9)$ & (2) $p=.02 ; d=0.14$ \\
\hline & AnT & $41.7(3.7)$ & $39.7(3.8)$ & $41.2(3.6)$ & \\
\hline & Maximal & $38.8(1.7)$ & $37.6(2.3)$ & $39.4(1.6)$ & \\
\hline \multirow[t]{3}{*}{$\mathrm{FeO}_{2}(\%)$} & AT & $16.2(0.1)$ & $16.4(0.1)$ & $16.2(0.1)$ & \\
\hline & $A n T$ & $16.4(0.2)$ & $16.5(0.3)$ & $16.4(0.2)$ & \\
\hline & Maximal & $16.8(0.3)$ & $16.8(0.3)$ & $16.8(0.3)$ & \\
\hline \multirow[t]{3}{*}{$\mathrm{FeCO}_{2}(\%)$} & AT & $4.3(0.1)$ & $4.1(0.1)$ & $4.3(0.1)$ & \\
\hline & AnT & $4.4(0.1)$ & $4.2(0.2)$ & $4.3(0.2)$ & \\
\hline & Maximal & $4.1(0.2)$ & $4.1(0.2)$ & $4.3(0.2)$ & \\
\hline
\end{tabular}

AT aerobic threshold (moderate exercise intensity), AnT anaerobic threshold (high exercise intensity), PETO ${ }_{2}$, end-tidal partial pressure of oxygen, $P E T C O_{2}$ end-tidal partial pressure of carbon dioxide, $\mathrm{FeO}_{2}$ expired fraction of $\mathrm{O}_{2}, \mathrm{FeCO}_{2}$ expired fraction of $\mathrm{CO}_{2},(1)$, statistically significant differences between olive oil and palm oil; (2), statistically significant differences between olive oil and placebo 

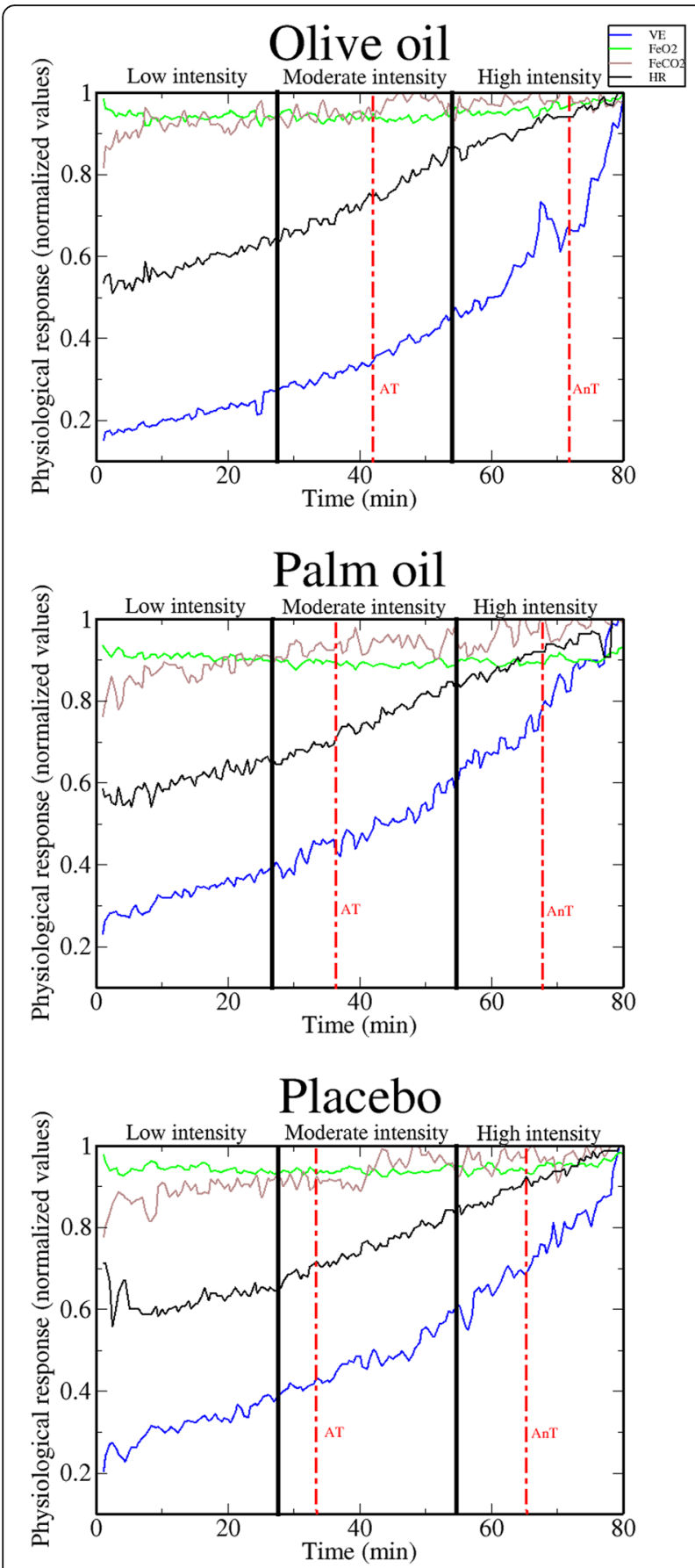

Fig. 1 Representation of the selected physiological variables in one single participant during the three dietary supplementations (olive oil, palm oil, and placebo). Each test was divided into three equal sections (low, moderate, and high intensity). The vertical red doted lines indicate the occurrence of aerobic and anaerobic thresholds. Note that a reduction in VE was observed at moderate intensity under olive oil supplementation. AT, aerobic threshold; AnT, anaerobic threshold

increases the levels of proinflammatory cytokines and reactive oxygen species (ROS) production. Thus, olive oil supplementation could have increased the antioxidant capacity while performing at moderate exercise intensity, reducing the negative effects of ROS accumulation [25]. A feasible explanation for the absence of differences in CRC among dietary supplementations at low and high intensity, might stem from the fact that markers of lipid peroxidation seem to be lower during mild and high intensity compared to moderate exercise [26]. The concentration of lipid peroxidation markers at low and high intensities was not probably enough to impair antioxidant capacity and, therefore, ROS clearance did not occur at a high enough rate to affect cardio-respiratory function. As a result, the antioxidant and anti-inflam matory effects of olive oil could be detected through CRC solely during moderate intensity exercise. Another explanation for the lack of differences at low and high intensity might be related to the Fat ${ }_{\max }$ zone [27], which seems to range from 40 to $75 \% \mathrm{VO}_{2 \max }$ [28]. The cutting points delimiting the moderate intensity interval in the current study were located at $40.29+10.57$ and $70.81+7.08 \% \mathrm{VO}_{2 \text { peak }}$. Thus, the previously observed slight effect of olive oil on fat oxidation [7], could have been magnified at moderate intensity (i.e., Fat ${ }_{\max }$ ), and this might have been detected by CRC analysis.

As depicted in Table 2, while comparing the projections of the selected cardiorespiratory variables onto $\mathrm{PC}_{1}$ at moderate intensity, VE projection was shown to be significantly higher in olive oil compared to palm oil supplementation. This means that the increment in the degree of CRC observed under olive oil supplementation was mainly provoked by a change in VE behaviour (i.e., a decrease in the absolute values of VE while performing at moderate intensity and at AT; see Fig. 1 and Table 2), which subsequently led to an increase in co-variation between VE and the other cardiorespiratory variables. The decreased VE under olive oil supplementation could be related to the high content in polyphenols [5], specifically, hydroxytyrosol [29], which may have some peripheral effects on mitochondrial function. As detailed in Hao et al., [29] relatively low doses of hydroxytyrosol increase the expression of all mitochondrial respiratory chain complexes, including ATP synthase, and stimulates mitochondrial biosynthesis pathway. This fact could let to an improvement of central control of VE and, thus, a reduction in ventilatory demands and an increase in ventilatory efficiency (i.e., a reduced VE for the same workload). In contrast, since palm oil and placebo supplementations contained no polyphenols [30], participants probably presented higher ventilatory demands. Accordingly, at moderate intensity (i.e., at AT), $\mathrm{PETO}_{2}$ was lower and $\mathrm{PETCO}_{2}$ was higher under olive oil, compared to palm oil supplementation (Table 3). Although VE and $\mathrm{PETO}_{2}$ were lower and $\mathrm{PETCO}_{2}$ was higher under olive oil, compared to both palm oil and placebo supplementations at moderate intensity, the reduced 
sample size of this study can probably explain why statistically significant differences were only found between olive oil and palm oil supplementations, but not with respect to placebo conditions.

In agreement with Boss et al. [7] and Capó et al. [6, 8], athletic performance (measured through exercising time) was not altered by olive oil supplementation, probably because the improvement on CRC observed in the current study was not strong enough to positively affect performance. Since changes associated to olive oil supplementation seem to be not only happening at cellular or subcellular level but also at systemic levels, and given that $\mathrm{CRC}$ is a coordinative variable which might be more sensitive to exercise and dietary supplementations than commonly registered physiological and performance variables, the tracking of changes in CRC may contribute to shed light on other unclear questions regarding the effectiveness of different fatty acid supplementations, such as omega-3 polyunsaturated fatty acids [31] or conjugated linoleic acid [32]. Further research should be conducted to provide an accurate picture of the effective ness of some controversial dietary supplementations throughout CRC evaluation, such as the physiological mechanisms underlying the impact of beetroot juice supplementation on cardiorespiratory function, in highly -trained endurance athletes [33].

Our findings should be discussed in the light of our methodological limitations. First, oxygen and $\mathrm{CO}_{2}$ content and air flow rate were monitored using a low frequency (i.e., every $30 \mathrm{~s}$ ), and continuous blood pressure monitoring could not be provided in this study. However, the findings obtained in the current research (i.e., high sensitivity and responsiveness of CRC, number of PCs, PC projections) are in full accordance and reinforce those published previously, where respiratory gas exchange was recorded breath by breath and blood pressure was monitored continuously [10, 11]. This means that CRC might be also studied using lower sampling rates when tests have longer duration and provide enough data sets. Second, since inflammatory and lipid peroxidation markers could not be assessed in this research, we cannot guarantee that the changes observed in CRC under olive oil supplementation were provoked by physiological adjustments at this level. Therefore, further research is warranted analysing CRC together with inflammatory and oxidation markers to confirm this hypothesis.

\section{Conclusions}

In conclusion, the supplementation with $25 \mathrm{ml}$ of extra virgin olive oil increased CRC during a progressive walking test while being performed at moderate intensity, although it did not change performance and other physio logical markers. CRC analysis appears as a sensitive tool to investigate the physiological and performance effects of dietary supplementations.

\section{Abbreviations \\ AnT: Anaerobic threshold; AT: Aerobic threshold; CRC: Cardiorespiratory coordination; $\mathrm{FeCO}_{2}$ : Expired fraction of $\mathrm{CO}_{2} ; \mathrm{FeO}_{2}$ : Expired fraction of $\mathrm{O}_{2}$; HR: Heart rate; $\mathrm{PC}_{1}$ : First principal component; $\mathrm{PCA}$ : Principal components analysis; $\mathrm{PETCO}_{2}$ : End-tidal partial pressure of carbon dioxide; $\mathrm{PETO}_{2}$ : End- tidal partial pressure of oxygen; ROS: Reactive oxygen species; VE: Ventilation; $\mathrm{VO}_{2 \text { max }}$ : Maximal oxygen uptake; $\mathrm{VO}_{2 \text { peak: }}$ Peak oxygen uptake}

\section{Acknowledgements}

The authors thank the participants for their dedication to the study.

Funding

Not applicable.

\section{Availability of data and materials}

The datasets used and/or analysed during the current study are available from the corresponding author on reasonable request.

\section{Authors' contributions}

$\mathrm{SG}, \mathrm{NB}, \mathrm{RH}$, and CJ conceived the paper and jointly drafted and reviewed the content; CJ and LE worked on acquisition of the data; LE worked on the active and placebo supplements; $\mathrm{RH}$ conceived the approach to data analysis. Since LE and SG contributed equally to this research, both can be considered as first authors. All authors read and approved the final manuscript.

\section{Ethics approval and consent to participate}

Participants read the study's description and risks and signed an informed consent before taking part in the study.

All experimental procedures were approved by the Bio-ethics commission of Barcelona University

\section{Consent for publication}

Not applicable.

\section{Competing interests}

The authors declare that they have no competing interests.

\section{Publisher's Note}

Springer Nature remains neutral with regard to jurisdictional claims in published maps and institutional affiliations.

\section{Author details}

${ }^{1}$ FoodLab, Faculty of Health Sciences, Universitat Oberta de Catalunya, Avda. del Tibidabo, 39-43, 08035 Barcelona, Spain. ${ }^{2}$ Department of Physiological Sciences, School of Medicine, Universitat de Barcelona (UB), C. Feixa Llarga, s/ n, Hospitalet de Llobregat 08907, Spain. ${ }^{3}$ Complex Systems in Sport, University School of Health and Sport (EUSES), University of Girona, C. Francesc Macià 65, 17190 Salt, Spain. ${ }^{4}$ Complex Systems in Sport, Institut Nacional d'Educació Física de Catalunya (INEFC), Universitat de Barcelona, Avda. de I'Estadi 12-22, 08038 Barcelona, Spain. ${ }^{5}$ Faculty of Physical Education, Sport and Health, Ss Cyril and Methodius University of Skopje, Zeleznicka BB, 1000 Skopje, Republic of Macedonia.

Received: 24 September 2018 Accepted: 19 February 2019

Published online: 01 March 2019

\section{References}

1. Guasch-Ferre M, Hu FB, Martinez-Gonzalez MA, Fito M, Bullo M, Estruch R, et al. Olive oil intake and risk of cardiovascular disease and mortality in the predimed study. BMC Med. 2014;12:78.

2. Martinez-Gonzalez MA, Toledo E, Aros F, Fiol M, Corella D, Salas-Salvado J, et al. Extravirgin olive oil consumption reduces risk of atrial fibrillation: the PREDIMED trial. Circulation. 2014;130:18-26.

3. García-Gavilán JF, Bullo M, Canudas S, Martínez-González MA, Estruch R, Giardina S, et al. Extra virgin olive oil consumption reduces the risk of osteoporotic fractures in the PREDIMED trial. Clin Nutr. 2018;37:329-35. 
4. Cicerale S, Lucas LJ, Keast RSJ. Antimicrobial, antioxidant and antiinflammatory phenolic: activities in extra virgin olive oil. Curr Opin Biotechnol. 2012;23:129-35.

5. Gorzynik-Debicka M, Przychodzen P, Cappello F, Kuban-Jankowska A, Marino Gammazza A, Knap N, et al. Potential health benefits of olive oil and plant polyphenols. Int J Mol Sci. 2018;19:547.

6. Capó X, Martorell M, Busquets-Cortés C, Sureda A, Riera J, Drobnic F, et al. Effects of dietary almond- and olive oil-based docosahexaenoic- and vitamin E-enriched beverage supplementation on athletic performance and oxidative stress markers. Food Funct. 2016;7:4920-34.

7. Boss A, Lecoultre V, Ruffieux C, Tappy L, Schneiter P. Combined effects of endurance training and dietary unsaturated fatty acids on physical performance, fat oxidation and insulin sensitivity. Br J Nutr. 2010;103: 1151-79.

8. Capó X, Martorell M, Sureda A, Riera J, Drobnic F, Tur JA, et al. Effects of almond- and olive oil-based docosahexaenoic- and vitamin E-enriched beverage dietary supplementation on inflammation associated to exercise and age. Nutrients. 2016;8:1-18.

9. Braakhuis AJ, Hopkins WG. Impact of dietary antioxidants on sport performance: a review. Sports Med. 2015;45:939-55.

10. Balagué N, González J, Javierre C, Hristovski R, Aragonés D, Álamo J, et al. Cardiorespiratory coordination after training and detraining. A principal component analysis approach. Front Physiol. 2016;7:35.

11. Garcia-Retortillo S, Javierre C, Hristovski R, Ventura JL, Balagué N. Cardiorespiratory coordination in repeated maximal exercise. Front Physiol. 2017;8:387.

12. O'Leary T, Collett J, Howells K, Morris MG. High but not moderate-intensity endurance training increases pain tolerance: a randomised trial. Eur J Appl Physiol. 2017;117:2201-10.

13. Bartsch RP, Schumann AY, Kantelhardt JW, Penzel T, Ivanov PC. Phase transitions in physiologic coupling. Proc Natl Acad Sci U S A. 2012;109: 10181-6.

14. Ivanov PC, Liu KK, Bartsch RP. Focus on the emerging new fields of network physiology and network medicine. 2016. New J Phys. 2016;18:100201.

15. Schulz S, Adochiei FC, Edu IR, Schroeder L, Costin H, Bär K, et al. Cardiovascular and cardiorespiratory coupling analyses: a review. Philos Trans AMath Phys Eng Sci. 2013;371:20120191.

16. Latash ML. Synergy. New York. Oxford University Press; 1998.

17. Scholz JP, Schöner G. The uncontrolled manifold concept: identifying control variables for a functional task. Exp Brain Res. 1999;126:289-306.

18. Kelso JAS. Dynamic patterns: the self-organization of brain and behavior. Cambridge, MA: MIT Press Ltd; 1995.

19. Faul F, Erdfelder E, Lang AG, Buchner AG. *power 3: a flexible statistical power analysis program for the social, behavioral, and biomedical sciences. Behav Res Methods. 2007;39:175-91.

20. Weinbrenner T, Fitó M, Farré Albaladejo M, Saez GT, Rijken P, Tormos C, et al. Bioavailability of phenolic compounds from olive oil and oxidative/ antioxidant status at postprandial state in healthy humans. Drugs Exp Clin Res. 2004;30:207-12.

21. Denis DJ. Applied univariate, bivariate, and multivariate statistics. Hoboken, NJ: Wiley; 2016.

22. Jolliffe I. Principal component analysis. New York, NY: Springer; 2002.

23. Reinhard $U$, Müller $\mathbf{P H}$, Schmülling $R$. Determination of anaerobic threshold by the ventilation equivalent in normal individuals. Respiration. 1979;38:36-42.

24. Sallam N, Laher I. Exercise modulates oxidative stress and inflammation in aging and cardiovascular diseases. Oxidative Med Cell Longev. 2016;2016: 7239639.

25. Ceriello A, Taboga C, Tonutti L, Quagliaro L, Piconi L, Bais B, et al. Evidence for an independent and cumulative effect of postprandial hypertriglyceridemia and hyperglycemia on endothelial dysfunction and oxidative stress generation: effects of short- and long-term simvastatin treatment. Circulation. 2002;106:1211-8.

26. McClean C, Harris RA, Brown M, Brown JC, Davison GW. Effects of exercise intensity on postexercise endothelial function and oxidative stress. Oxidative Med Cell Longev. 2015;2015:723679.

27. Jeukendrup AE, Saris WH, Wagenmakers AJ. Fat metabolism during exercise: a review-part III: effects of nutritional interventions. Int J Sports Med. 1998; 19:371-9.
28. Achten J, Venables MC, Jeukendrup AE. Fat oxidation rates are higher during running compared with cycling over a wide range of intensities. Metabolism. 2003;52:747-52.

29. Hao J, Shen W, Yu G, Jia H, Li X, Feng Z, et al. Hydroxytyrosol promotes mitochondrial biogenesis and mitochondrial function in 3T3-L1 adipocytes. J Nutr Biochem. 2010;21:634-44.

30. Kien CL, Bunn JY, Ugrasbul F. Increasing dietary palmitic acid decreases fat oxidation and daily energy expenditure. Am J Clin Nutr. 2005;82:320.

31. Thota RN, Ferguson JJA, Abbott KA, Dias CB, Garg ML. Science behind the cardio-metabolic benefits of omega-3 polyunsaturated fatty acids: biochemical effects vs. clinical outcomes. Food Funct. 2018;9:3576-96.

32. Haghighatdoost F, Hariri M. Effect of conjugated linoleic acid supplementation on serum leptin concentration: a systematic review and meta-analysis. Endocr Metab Immune Disord Drug Targets. 2018;18:185-93.

33. Garnacho-Castaño MV, Palau-Salvà G, Cuenca E, Muñoz-González A, GarcíaFernández $\mathrm{P}$, Lozano-Estevan $\mathrm{M}$, et al. Effects of a single dose of beetroot juice on cycling time trial performance at ventilatory thresholds intensity in male triathletes. J Int Soc Sports Nutr. 2018;15:49.

\section{Ready to submit your research? Choose BMC and benefit from:}

- fast, convenient online submission

- thorough peer review by experienced researchers in your field

- rapid publication on acceptance

- support for research data, including large and complex data types

- gold Open Access which fosters wider collaboration and increased citations

- maximum visibility for your research: over $100 \mathrm{M}$ website views per year

At BMC, research is always in progress.

Learn more biomedcentral.com/submissions 